\title{
BMJ Open Association between N95 respirator wearing and device-related pressure injury in the fight against COVID-19: a multicentre cross-sectional survey in China
}

\author{
Qixia Jiang (D) , ${ }^{1}$ Yuxiu Liu, ${ }^{2}$ Siping Song, ${ }^{3}$ Wei Wei, ${ }^{3}$ Yuxuan Bai ${ }^{4}$
}

To cite: Jiang Q, Liu Y, Song S, et al. Association between N95 respirator wearing and device-related pressure injury in the fight against COVID-19: a multicentre cross-sectional survey in China. BMJ Open 2021;11:e041880. doi:10.1136/ bmjopen-2020-041880

- Prepublication history for this paper is available online. To view these files, please visit the journal online (http://dx.doi org/10.1136/bmjopen-2020041880).

QJ and YL contributed equally.

Received 23 June 2020 Revised 23 January 2021 Accepted 27 January 2021

Check for updates

(C) Author(s) (or their employer(s)) 2021. Re-use permitted under CC BY-NC. No commercial re-use. See rights and permissions. Published by BMJ.

${ }^{1}$ Department of Burns and Plastic Surgery, Jinling Hospital, Medical School of Nanjing University, Nanjing, China ${ }^{2}$ Department of Medical Statistics, Jinling Hospital, Nanjing Medical University, Nanjing, China

${ }^{3}$ Graduate department of Medical School, Nanjing University, Nanjing, China ${ }^{4}$ Clinical Academic Department, Zhejiang Top-medical Dressing Co., Ltd, Wenzhou, China

Correspondence to

Dr Qixia Jiang;

jiangqixia1963@163.com

\section{ABSTRACT}

Objectives To explore the association between N95 respirator wearing and device-related pressure injury (DRPI) and to provide a basis for protecting medical staff from skin injuries.

Design A cross-sectional, multicentre study.

Setting and participants Medical staff of 60 hospitals were selected from 145 designated medical institutions located in the epidemic area where the patients with COVID-19 were treated in China.

Results In total, 1761 respondents wore N95 respirators (use alone 20.8\%; combination use $79.2 \%$ ), and the prevalence of DRPI was $59.2 \%$ (95\% Cl 56.93 to 61.53$)$. A daily wearing time of $>4$ hours (OR $1.62,95 \% \mathrm{Cl}$ 1.11 to 2.35), wearing a N95 respirator in combination with goggles both with the presence of sweating (OR $13.40,95 \% \mathrm{Cl} 7.34$ to 23.16 ) and without the presence of sweating (OR $0.80,95 \% \mathrm{Cl} 0.56$ to 1.14) and wearing only a N95 respirator with the presence of sweating (OR $9.60,95 \% \mathrm{Cl} 7.00$ to 13.16 ) were associated with DRPI. A correspondence analysis indicated that if there was no sweating, regardless of whether the N95 respirator was worn by itself or in combination with goggles, singlesite DRPI mainly occurred on the nose bridge, cheek and auricle. If there was sweating present, regardless of whether the $\mathrm{N} 95$ was worn by itself or in combination with goggles, multiple DRPI sites occurred more often on the face.

Conclusions The prevalence of DRPI among medical staff caused by N95 respirators was very high, which was mainly associated with a longer daily wearing time and interaction with sweating. The nasal bridge, cheeks and auricles were the primary protection locations found.

\section{INTRODUCTION}

Currently, the COVID-19 pandemic has spread to $>200$ countries, and 500 million people are infected; approximately 350000 people have been killed by the disease. The pandemic is becoming the largest public health emergency around the world. ${ }^{12}$ Early studies have indicated that patients with COVID-19 and those who are virus carriers could have
Strengths and limitations of this study

- The prevalence of device-related pressure injury (DRPI) due to wearing a N95 respirator for doctors and nurses was $60.2 \%$ and $59.1 \%$, respectively.

- Daily wearing time was an independent risk factor for DRPI, and there was an interaction between wearing condition and sweating.

- If there was sweating, regardless of whether the N95 respirator was worn by itself or in combination with goggles, multiple sites of DRPI occurred more often on the face.

- The dependent variable and independent variable are further defined in the part of statistical analyses and the corresponding analysis is reformulated to make it more specific.

- It is difficult to observe and analyse prevention outcomes and the degree of sweating in medical staff who are wearing a $\mathrm{N} 95$ respirator.

been infected by human-to-human transmission. ${ }^{34}$ In every country, medical staff have become the most important pioneers in the fight against the COVID-19 pandemic. These individuals work hard daily for 8-12hours while wearing personal protective equipment (PPE), including protective face masks (N95 respirator use by itself or combination with goggles), protective gowns, latex gloves and shoes. The N95 respirator, as a primary piece of PPE, plays an important role in protecting doctors and nurses from becoming infected with the disease ${ }^{56}$ and is considered a part of the strict occupational protection measures for medical staff who are fighting COVID$19 .^{7}$ Therefore, it is recommended that all medical staff working in the frontline against COVID-19 wear a N95 respirator in China. ${ }^{5,6}$

Unfortunately, approximately $80 \%$ of N95 respirator users reported multiple-sites pressure injuries on their face during working 
on the front lines of COVID-19, also known as devicerelated pressure injury (DRPI) ${ }^{89}$ Previously, DRPI mostly occurred in critically ill patients ${ }^{9-11}$; however, now DRPI is becoming a new occupational injury for medical staff worldwide who wear a N95 respirator while fighting against COVID-19. Therefore, we organised a multicentre, cross-sectional survey to understand the association between N95 respirator wearing and DRPI and to provide a basis for frontline medical staff in regard to protecting their skin from occupational injuries while wearing N95 respirators both in China and in other countries around the world.

\section{METHODS \\ Study design}

A cross-sectional multicentre study was performed aimed at evaluating the prevalence of DRPI and exploring the association between wearing a N95 respirator and DRPI to help provide a basis for preventive measures in regard to medical staff.

\section{Materials and samples}

The sample size was determined based on $80 \%$ of the medical staff who reported facial injuries while wearing N95 respirators. Considering a tolerated absolute error of $2 \%$ and a CI of $95 \%$, the sample size calculated was 1537 participants. Bearing in mind that $5 \%$ of the returned questionnaires may be invalid, a total of 1618 participants were needed.

The inclusion criteria were as follows: (1) medical staff who worked in an environment with either a moderate risk of exposure to COVID-19 (emergency departments and screening clinics where suspected cases are treated) or a high risk of exposure to COVID-19 (intensive care units (ICUs) and wards in designated medical institutions located in the epidemic area where patients with COVID-19 were treated $)^{12} 13$ and wore only a N95 respirator or wore one in combination with other PPE; (2) those aged 20-60 years, regardless of gender and (3) those who voluntarily participated.

The exclusion criteria were as follows: (1) medical staff who did not wear a N95 respirator or who used other alternatives; and (2) questionnaires that were incomplete or invalid.

\section{Data collection}

We designed a data collection questionnaire consisting of 10 items after consulting the relevant literature and guidelines, ${ }^{811}$ and we received feedback about the questionnaire from frontline medical staff in Wuhan. The data collection included three aspects of information: four items related to general demographic information (gender, age, current working position and occupation); three items related to the wearing of a N95 respirator (single use or in combination with goggles, wearing time per day and with or without sweating) and three items related to DRPI information (when the injury happened, anatomical location, pressure injury classification). ${ }^{8}$ Before the formal investigation, 30 medical staff members were selected for a pilot survey. It took $3-4$ min for each person to complete the questionnaire. One hundred per cent of these respondents thought that the questionnaire was clear and easy to answer.

We uploaded the questionnaire on the Questionnaire Star survey tool and disseminated it to groups of medical teams providing support to Wuhan in infectious disease departments, isolation wards and ICUs via the WeChat social platform on 8 February 2020. Before the survey was disseminated, the inclusion and exclusion criteria and the survey objective used for the recruitment of participants was openly available online in China. All the participants voluntarily provided their informed consent. The survey was completed anonymously. The privacy and confidentiality of the study participants were also strictly maintained. The participants voluntarily used their mobile phone to answer and submit the questionnaire online until 22 February 2020.

\section{Research quality control}

We provided single-choice and multiple-choice responses to the participants in order to answer quickly and accurately. The classification of DRPI depended on the staging system set forth in the 2019 International Guide for Prevention and Treatment of Pressure Ulcer/Injury, that is, stages 1, 2, 3, 4, deep tissue injury or unstageable. ${ }^{8}$ All the data were output gathered from the Questionnaire Star website. Then, two researchers checked each questionnaire to estimate if it was completed and if the answers were logical; finally a database was established for the statistical analysis.

\section{Patient and public involvement}

No patients were involved.

\section{Statistical analyses}

All analyses were based on complete data cases. The Shapiro-Wilk test was used to test the normality of the continuous variables. Continuous variables were presented as the mean with the SD or as the median with the IQR where necessary. Categorical variables were described as frequencies and percentages. Student's t-tests or Mann-Whitney $\mathrm{U}$ tests were used to compare the differences between the groups for continuous or ordinal variables. Pearson's $\chi^{2}$ tests or Fisher's exact tests were used for comparing categorical variables, where applicable. First, the univariate analysis was performed with DRPI as the dependent variable ( $0=$ none, $1=$ yes $)$, and with the wearing condition $(1=\mathrm{N} 95$ respirator, $2=\mathrm{N} 95$ respirator with goggles), daily wearing time (DWT) ( $1=\mathrm{DWT} \leq 4$ hours, $2=\mathrm{DWT}>4$ hours), sweating $(0=$ none, $1=$ yes $)$, gender $(0=$ male, $1=$ female $)$, age $(1=<35$ years, $2=\geq 35$ years) and occupation (1=doctors, $2=$ nurses) as independent variables. Second, the binary logistic regression analyses were used to evaluate the influence of independent risk factors. Those variables with a probability 
value of $\mathrm{p}<0.10$ in the univariate analysis were entered into the multivariate logistic regression model, which used the backward method to establish whether they were independently associated with DRPI. Interaction analysis was employed to explore the risk characteristics of wearing only N95 respirators or wearing them in combination with other PPE on outcomes of DRPI. If there were interactions between the factors, we analysed their combinations to obtain the OR and $95 \%$ CI values of different combinations; if there were no interactions, we analysed the factors separately as independent variables. Based on all subjects with DRPI, a contingency table of different behaviour characteristics related to wearing PPE and the anatomical locations of the reported DRPI was constructed, and the associations between these factors were evaluated by correspondence analysis. A p value of 0.05 or less was considered to indicate statistical significance. All statistical analyses were performed using SPSS V.22.0 for Windows (SPSS, Chicago, Illinois, USA).

\section{RESULTS}

Characteristics of device-related pressure injury in medical staff wearing $\mathrm{N} 95$ respirators

In this survey, an online mode was adopted, and the possibility of missing data caused by no responses of the respondents was small. All analyses were performed based on complete data cases. In total, 1800 respondents participated from 60 hospitals of 145 designated medical institutions located across 12 provinces in China, and 39 respondents were excluded for not wearing a N95 respirator. Finally, 1761 respondents who wore N95 respirators were included. Of them, 366 respondents $(20.8 \%)$ wore a N95 respirator only, and 1395 respondents $(79.2 \%)$ wore a N95 respirator plus goggles. The average DWT was $7.0 \pm 2.3$ hours, with $89.4 \%$ (1574 respondents) wearing the respirator for $>4$ hours and $62.2 \%$ (1096 respondents) reporting sweating. The respondents were $16.5 \%$ male (290 respondents) and $83.5 \%$ female (1471 respondents), and $13.4 \%$ of them were doctors (236 respondents), while $86.6 \%$ of them were nurses (1525 respondents). In total, there were 1043 cases with 2413 DRPI locations, and the total prevalence of DRPI was $59.2 \%$ (95\% CI 56.93 to 61.53$)$. The prevalence of those who reported wearing N95 respirators combined with goggles was higher than that of those who reported wearing only N95 respirators (62.9\% (95\% CI 61.40 to 66.59 ) vs $45.4 \%$ (95\% CI 41.30 to 51.60), $\mathrm{p}<0.001$ ); the prevalence for those wearing N95 respirators with reported sweating $(79.5 \%)$ was higher than that for those without reported sweating (30.3\%) $(\mathrm{p}<0.001)$; and the prevalence of those who reported wearing DWT $>4$ hours $(63.2 \%)$ was higher than that of those who reported wearing DWT $\leq 4$ hours $(37.4 \%)$ $(\mathrm{p}<0.001)$. The characteristics of DRPI in the medical staff who reported wearing a N95 respirator are shown in table 1.

\begin{tabular}{|c|c|c|c|c|}
\hline Characteristics & $\mathbf{N}$ & Prevalence of DRPI & $95 \% \mathrm{Cl}$ for prevalence & $P$ value \\
\hline Wearing condition & & & & $<0.001$ \\
\hline N95+goggles (n, \%) & 1395 & 877 (62.9) & 60.33 to 65.40 & \\
\hline N95 by itself (n, \%) & 366 & $166(45.4)$ & 40.25 to 50.46 & \\
\hline Daily wearing time & & & & $<0.001$ \\
\hline$>4$ hours & 1574 & $974(61.9)$ & 59.48 to 64.28 & \\
\hline$\leq 4$ hours & 187 & $69(37.4)$ & 29.98 to 43.81 & \\
\hline Sweating & & & & $<0.001$ \\
\hline Yes & 1096 & 876 (79.9) & 77.56 to 82.30 & \\
\hline No & 665 & $167(25.1)$ & 21.82 to 28.41 & \\
\hline Gender & & & & 0.181 \\
\hline Male & 290 & $182(62.8)$ & 57.19 to 68.32 & \\
\hline Female & 1471 & $861(58.5)$ & 56.01 to 61.05 & \\
\hline Age (years) & & & & 0.865 \\
\hline$<35$ & 1196 & $710(59.4)$ & 56.58 to 62.15 & \\
\hline$\geq 35$ & 565 & $333(58.9)$ & 54.88 to 62.99 & \\
\hline Occupation & & & & 0.752 \\
\hline Doctors & 236 & $142(60.2)$ & 53.92 to 66.42 & \\
\hline Nurses & 1525 & $901(59.1)$ & 56.61 to 61.56 & \\
\hline
\end{tabular}

DRPI, device-related pressure injury; DWT, daily wearing time; N95, N95 respirator; N95+goggles, N95 respirator in combination with goggles. 
Table 2 Multivariate logistic analyses of DRPI in medical staff wearing N95 respirator

\begin{tabular}{llll}
\hline Variables & OR & 95\% Cl for OR & P value \\
\hline Wearing condition and sweating & & & \\
N95+goggles with sweating & 13.40 & 7.34 to 23.16 & 0.001 \\
N95+goggles without sweating & 0.80 & 0.56 to 1.14 \\
N95 by itself with sweating & 9.60 & 7.00 to 13.16 \\
N95 by itself without sweating & 1.00 & Reference \\
Daily wearing time $>$ 4hours & 1.62 & 1.11 to 2.35 \\
\hline
\end{tabular}

DRPI, device-related pressure injury; N95+goggles, N95 respirator in combination with goggles.

Logistic regression analysis of device-related pressure injury caused by $\mathrm{N} 95$ respirator

The dichotomous variable of DRPI was regressed as a dependent variable (none $=0$, yes $=1$ ).

By entering those variables with a probability value of $p<0.10$ based on univariate analysis (in table 1) into a multivariate logistic regression model, the backward method was selected to establish whether the variables were independently associated with DRPI. An interaction analysis was employed to explore the risk characteristics of wearing either a N95 respirator plus goggles or only wearing a N95 respirator on the occurrence of DRPI, and the results indicated that DWT and reported sweating were associated with DRPI (the omnibus test of model coefficients was $\mathrm{p}<0.001$; the Hosmer-Lemeshow test for goodness of fit of the model was $\mathrm{p}=0.297$; $\mathrm{C}$-index $=0.78$ ); in addition, there was an interaction between wearing a N95 respirator and sweating (the omnibus test of model coefficients was $\mathrm{p}<0.001$; the Hosmer-Lemeshow test for goodness of fit of the model was $\mathrm{p}=0.250$; $\mathrm{C}$-index $=0.79$ ), but there was no interaction between wearing a N95 respirator and DWT (the omnibus test of model coefficients was $\mathrm{p}<0.001$; the Hosmer-Lemeshow test for goodness of fit of the model was $\mathrm{p}=0.381$; C-index $=0.78$ ). As an independent factor, DWT was analysed with interactive variables (wearing a N95 respirator plus goggles or wearing only a N95 respirator, both reported with and without sweating) in a multivariate logistic regression model. The omnibus test of model coefficients was $\chi^{2}=754.838(\mathrm{df}=4$, $\mathrm{p}<0.001)$. The Hosmer-Lemeshow test for goodness of fit of the model was $\chi^{2}=3.308(\mathrm{df}=4, \mathrm{p}=0.508)$. Approximately $82.1 \%$ of the participants could be classified correctly through the model. These results showed that the model fit the data well and could reasonably explain the relevant results. The results are shown in table 2 .

\section{Correspondence analysis of anatomical locations and N95 respirator}

In 1043 cases, the reported DRPI was mainly located on the nasal bridge, cheek and auricle, and there were two to three locations with coexisting features. Stage 1 (nonblanchable erythema) $(79.6 \%$ ) and stage 2 (partial thickness skin loss) $(19.4 \%)$ were the main categories. The staging and location distribution of the reported DRPI are shown in table 3.

A correspondence analysis of the 1043 cases with DRPI was conducted to analyse the results of the DRPI in different locations under the scenarios of N95 use with goggles or N95 use by itself, reported either with or without sweating, to determine the most easily injured anatomic locations and most prevalent causes. The $\chi^{2}$ value of this correspondence analysis was $229.678(\mathrm{p}<0.0001)$, which suggests highly significant associations. The proportion of inertia in the correspondence analysis indicated that

Table 3 Staging characteristics on locations of DRPI in medical staff wearing N95 respirator (cases of DRPI=1043)

\begin{tabular}{|c|c|c|c|c|c|}
\hline \multirow[b]{2}{*}{ Location } & \multicolumn{4}{|c|}{ Cases (proportion \%) } & \multirow{2}{*}{$\begin{array}{l}\text { Prevalence } \\
\text { Rate }(95 \% \mathrm{Cl})^{\star}\end{array}$} \\
\hline & Stage 1 & Stage 2 & Stage 3 & DTI & \\
\hline Nose bridge & $75(68.8)$ & $32(29.4)$ & $0(0.0)$ & $2(1.8)$ & 6.2 (5.06 to 7.32$)$ \\
\hline Auricle & $30(76.9)$ & $9(23.1)$ & $0(0.0)$ & $0(0.0)$ & 2.2 (1.53 to 2.90$)$ \\
\hline Nose+cheeks & $169(82.0)$ & $35(17.0)$ & $1(0.5)$ & $1(0.5)$ & 11.7 (10.20 to 13.20$)$ \\
\hline Cheeks+auricle & $68(86.1)$ & $10(12.7)$ & $0(0.0)$ & $1(1.3)$ & 4.5 (3.52 to 5.45$)$ \\
\hline Nose+cheeks+auricle & 359 (81.8) & $76(17.3)$ & $1(0.2)$ & $3(0.7)$ & 24.9 (22.91 to 26.95$)$ \\
\hline Total & $830(79.6)$ & $202(19.4)$ & $3(0.3)$ & $8(0.7)$ & \\
\hline
\end{tabular}

*These rates are the prevalence rate of DRPI for specific locations.

DRPI, device-related pressure injury; DTI, deep tissue injury. 


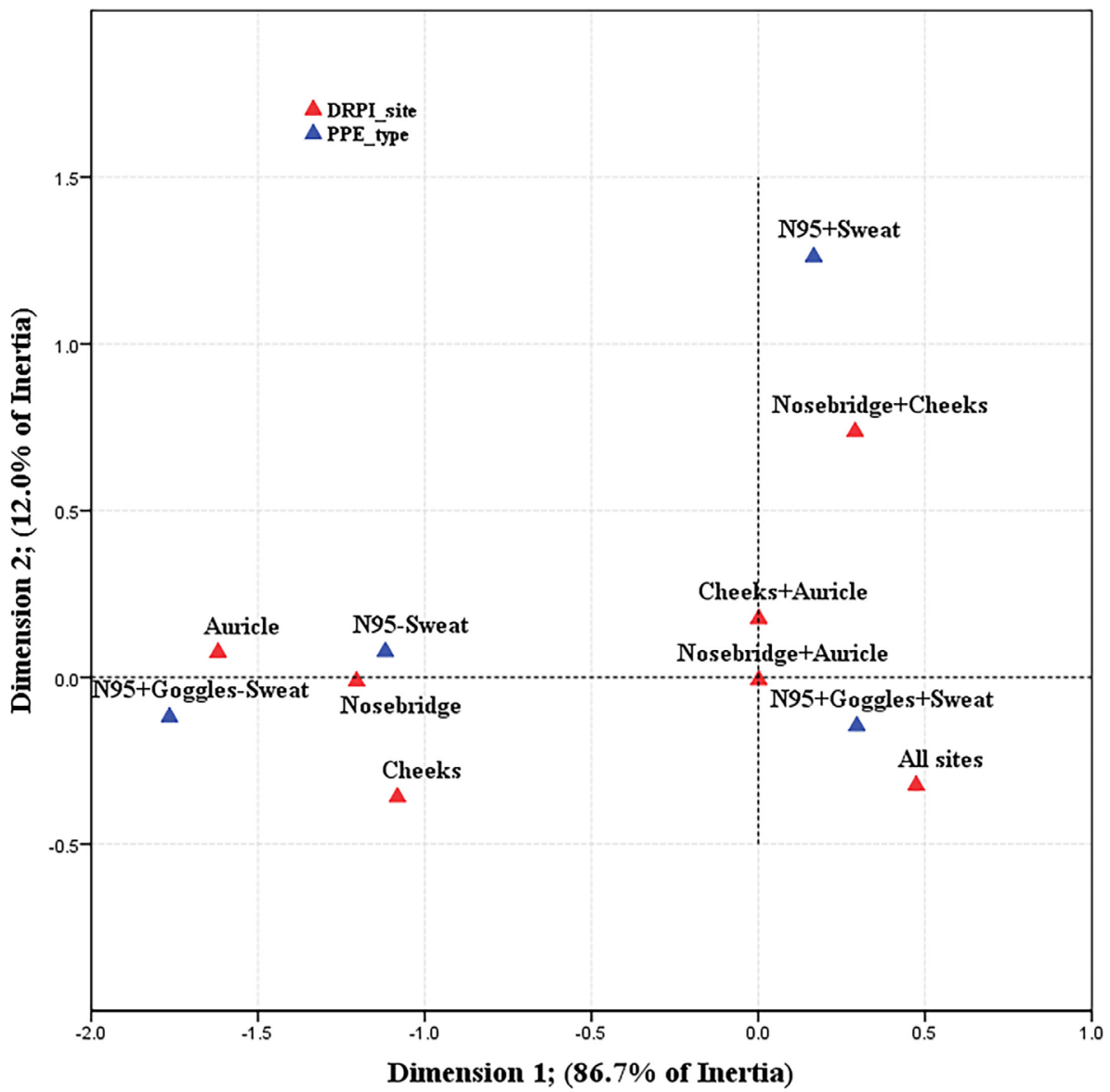

Note: N95-Sweat $=$ N95 worn by itself without sweating $\mathrm{N} 95+$ Sweat $=\mathrm{N} 95$ worn by itself with sweating

$\mathrm{N} 95+$ goggles + sweat $=$ N95 worn in combination with goggles and with sweating

N 95+goggles-sweat $=$ N95 worn in combination with goggles but without sweating

Figure 1 Correspondence analysis of personal protective equipment (PPE) wearing condition, sweating and sites of devicerelated pressure injury (DRPI).

the first dimension could explain $86.7 \%$ of the total variation, the second dimension could explain $12.0 \%$ and the sum of both can explain $98.7 \%$ of the variation. Therefore, it was reasonable to carry out the correspondence analysis. The results are shown in figure 1 .

\section{DISCUSSION}

The study significance of association between N95 respirator wearing and device-related pressure injury

Since December 2019, the COVID-19 pandemic has caused many problems worthy of global attention. Unexpected skin injuries due to wearing PPE in the working environment have become a topic of concern. ${ }^{1415}$ Therefore, China's government released a 'Notice on Further Strengthening the Protection of Medical Personnel During Epidemic Prevention and Control, ${ }^{16}$ calling for the protection of medical staff's health and safety, including the prevention of occupational injuries. Medical staff also reported that their results of pressure injuries were partially improved by improving PPE wearing technique. ${ }^{15}$ The issue of how to protect medical staff from occupational injuries is an important problem to be solved in the prevention and control of COVID-19. Therefore, we believed that it was necessary to explore the association between DRPI and using a N95 respirator, as such knowledge would have positive implications for China and other countries, especially in regard to the advanced prevention of occupational skin injuries among medical staff during responses to public health emergencies in the future.

\section{The characteristics of device-related pressure injury in medical staff wearing $\mathrm{N} 95$ respirators}

Our research showed that $89.4 \%$ of the 1761 respondents had a reported N95 respirator DWT of $>4$ hours. More than $80 \%$ of the medical staff reported that facial pressure began within 2 hours of wearing, mainly in the areas of the nose bridge and cheeks; the longer the N95 respirator was worn, the more severe the reported pain and discomfort were, which explains why the DRPI prevalence in the group who reported wearing the N95 respirator $\geq 4$ hours was significantly higher than that in the group wearing the N95 respirator <4hours $(61.9 \%$ vs $37.4 \%$, 
$\mathrm{p}<0.001)$. The characteristics of DRPI in medical staff who reported wearing only a N95 respirator compared with those who reported wearing a respirator in combination with goggles indicated that the prevalence of DRPI between gender, age and occupation (doctors and nurses) was not significant (table 1). One possible cause was that 1143 respondents $(64.9 \%)$ worked in an environment with a high exposure risk, with similar proportions of age, gender and occupation, working intensity and DWT. Interestingly, we found that the cases of DRPI in the N95 respirator-wearing condition (either by itself or in combination with goggles), DWT (either $\geq 4$ hours or $<4$ hours), both with and without sweating, was significant (table 1, $\mathrm{p}<0.001$ ); this outcome suggests that the DRPI induced by the N95 respirator may be associated with the interaction of DWT and sweating. These problems deserve further discussion.

\section{Analysis of the related factors and interactions of device- related pressure injury caused by the $\mathbf{N 9 5}$ respirator}

The logistic model was also fitted with all factors present in the univariable analysis. If the risk variables that showed statistical significance were consistent in the backward method, then the risk factors were confirmed more robustly. The results showed that there was no interaction between DWT and the wearing condition of the N95 respirator $(\mathrm{p}=0.489)$; however, there was an interaction between the wearing condition of the N95 respirator and sweating $(\mathrm{p}<0.001)$. A multivariate logistic analysis of DRPI showed that DWT and sweating were independent factors $(\mathrm{p}<0.05-$ 0.001) (table 2), which suggests that a DWT of $>4$ hours would increase the risk of DRPI (OR 1.62, 95\% CI 1.11 to 2.35), regardless of whether the N95 respirator was worn by itself or in combination with goggles. Setting the group of individuals who reported wearing only a N95 respirator without sweating as the reference group, the risk of DRPI caused by wearing a N95 respirator in combination with goggles plus reported sweating reached the highest value (OR 13.40, 95\% CI 7.34 to 23.16), followed by wearing only a N95 respirator with reported sweating (OR 9.60, 95\% CI 7.00 to 13.16) (table 2). This outcome suggests that sweating may change the skin's microclimate and increase the friction coefficient between the skin and the N95 respirator, while the combined effect of the N95 respirator's direct compression, friction and changed microclimate may reduce the skin's tolerance and make the skin more vulnerable to mechanical forces, such that DRPI is prone to occur. This principle of action is in line with the new conceptual framework of pressure injury updated in 2014. ${ }^{17}$

Interestingly, medical staff reported that wearing a N95 respirator combined with goggles did not have an increased risk of DRPI if without sweating. Therefore, preventing or managing sweating is expected to be an effective way to prevent DRPI.

Correspondence analysis of the anatomical locations of device-related pressure injury caused by the $\mathbf{N 9 5}$ respirator To further understand the role of the N95 respirator and sweating in the occurrence of DRPI, we carried out a correspondence analysis. The outcome of the correspondence analysis of the different behaviour characteristics related to wearing PPE and the anatomical locations of DRPI showed the degrees of separation in the row and column categories of the corresponding contingency table. There was a clear separation between the PPE characteristics and the DRPI locations. The proximity among the categories of the different variables revealed trends of association. Specifically, the results indicated that if there was no sweating present, regardless of whether the N95 respirator was worn by itself or in combination with goggles, single-site DRPI mainly occurred on the nose bridge, cheek and auricle (see the left area in figure 1); however, if there was a N95 respirator worn in combination with goggles plus reported sweating, multiple DRPI locations occurred on the face (see the lower right area in figure 1 ). When medical staff wore only a N95 respirator and reported sweating, DRPI was mainly found on the nose bridge and cheek (see the upper right area in figure 1). These injury occurrence characteristics clearly reflect the clinical characteristics and possibly indicate that multiple injuries in these areas are caused by wearing a N95 respirator. This means that the nasal bridge, cheeks and auricles should be the primary locations that need protection. We suggest that silicone foam dressings be used prior to putting on a N95 respirator to protect the cheek, auricle and nose bridge, dependent on the recommendation of the Prevention and Treatment of Pressure Ulcers/Injuries: Clinical Practice Guideline in 2019. ${ }^{8}$ In practice, silicone foam dressings can effectively reduce pressure and absorb sweat to protect the skin from injuries. ${ }^{18}$ The auricle is an extremely weak area of the human body, and DRPI easily occurs on in this area due to the pressure of the N95 respirator's straps. Thus, we suggest that the N95 respirator's straps should be tied to either the top of the head or one's hair, which could effectively prevent occupational injuries on auricles. ${ }^{19} 20$

\section{Limitations}

There were several limitations of the current study. First, because of the pandemic emergency, it is difficult to observe and analyse prevention outcomes and the degree of sweat in medical staff who wear a N95 respirator. Second, it is also difficult to analyse and compare the effect of wearing a N95 respirator either by itself or in combination with goggles on the different stage of DRPI. In summary, these issues leave us with opportunities for further research.

\section{CONCLUSION}

The prevalence of DRPI among medical staff caused by wearing a N95 respirator is very high and is mainly associated with both a longer DWT and the interaction of wearing a N95 respirator either by itself or in combination with goggles and the presence of sweating. Prevention practices should focus on the combined action of multiple factors. The results of the current study provide 
an important basis for strengthening skin protection and preventing occupational injuries among medical personnel who are fighting against COVID-19.

Acknowledgements The authors would like to thank the coordinators and medical staff of the participating hospitals, and the technical experts at AJE who editing for proper writing, English language, grammar, punctuation and spelling.

Contributors QJ, as a leader of skin and wound care in Jinling Hospital, Medical School of Nanjing University. She is responsible for designing questionnaire and organising survey and quality control, drafting the article and revising it. YL, as a director of Department of Medical Statistics in Jinling Hospital, Nanjing Medical University. He is responsible for checking design protocol and statistical analysis. SS assists in checking and setting up databases, searches literature and revises paper. WW and YB are responsible for checking data and setting up databases.

Funding This study was funded by Wang Zhengguo Foundation for Traumatic Medicine (WZGF20200101) and Military Medical Service Special Project (20WQ027).

Competing interests None declared.

Patient and public involvement Patients and/or the public were not involved in the design, or conduct, or reporting, or dissemination plans of this research.

Patient consent for publication Not required.

Ethics approval This study was approved by the medical ethics committee of Jingling Hospital, Medical School of Nanjing University (2019NZGKJ-025). Participants have given informed consent before taking part in this study.

Provenance and peer review Not commissioned; externally peer reviewed.

Data availability statement Data are available on reasonable request. Data may be obtained on reasonable request from corresponding author and are not publicly available.

Open access This is an open access article distributed in accordance with the Creative Commons Attribution Non Commercial (CC BY-NC 4.0) license, which permits others to distribute, remix, adapt, build upon this work non-commercially, and license their derivative works on different terms, provided the original work is properly cited, appropriate credit is given, any changes made indicated, and the use is non-commercial. See: http://creativecommons.org/licenses/by-nc/4.0/.

ORCID iD

Qixia Jiang http://orcid.org/0000-0003-0017-4353

\section{REFERENCES}

1 National Health Commission of the People's Republic of China. Law of the people's republic of china on prevention and treatment of infectious diseases [EB/OL], 2020. Available: http://www.nhc.gov.cn/ jkj/s7915/202001/e4e2d5e6f01147e0a8df3f6701d49f33.shtml

2 National Health Commission of the People's Republic of China. The management of COVID-19 prevention and control (The fifth edition) [EB/OL], 2020. Available: http://www.nhc.gov.cn/jkj/s3577/202002/ a5d6f7b8c48c451c87dba14889b30147/files/3514cb996ae24e2faf65 953b4ecd0df4.pdf

3 Guan W-J, Ni Z-Y, Hu Y, et al. Clinical characteristics of coronavirus disease 2019 in China. N Engl J Med 2020;382:1708-20.

4 Zhu N, Zhang D, Wang W, et al. A novel coronavirus from patients with pneumonia in China, 2019. N Engl J Med 2020;382:727-33.

5 Loeb M, Dafoe N, Mahony J, et al. Surgical mask vs N95 respirator for preventing influenza among health care workers: a randomized trial. JAMA 2009;302:1865-71.

6 Smith JD, MacDougall CC, Johnstone J, et al. Effectiveness of N95 respirators versus surgical masks in protecting health care workers from acute respiratory infection: a systematic review and metaanalysis. CMAJ 2016;188:567-74.

7 Wang X, Pan Z, Cheng Z. Association between 2019-nCoV transmission and N95 respirator use. J Hosp Infect 2020;105:104-5.

8 European Pressure Ulcer Advisory Panel, National Pressure Injury Advisory Panel and Pan Pacific Pressure Injury Alliance. Prevention and treatment of pressure Ulcers/Injuries: clinical practice guideline. The International guideline. 3rd Edition. Emily Haesler (ED). EPUAP/ NPIAP/PPPIA, 2019. Available: http://www.internationalguideline. $\mathrm{com} /$

9 Barakat-Johnson M, Barnett C, Wand T, et al. Medical device-related pressure injuries: an exploratory descriptive study in an acute tertiary hospital in Australia. J Tissue Viability 2017;26:246-53.

10 Barakat-Johnson M, Lai M, Wand T, et al. The incidence and prevalence of medical device-related pressure ulcers in intensive care: a systematic review. J Wound Care 2019;28:512-21.

11 Jackson D, Sarki AM, Betteridge R, et al. Medical device-related pressure ulcers: a systematic review and meta-analysis. Int $J$ Nurs Stud 2019;92:109-20.

12 Chinese Center for Disease Control and Prevention. Technical guidelines on the selection and use of anti-COVID-19 respirator for different populations, 2020. Available: http://www.nhc.gov.cn/jkj/ s7916/202002/485e5bd019924087a5614c4f1db135a2.shtml

13 National Health Commission of the People's Republic of China. Guidelines on medical protective products during prevention and control of viral pneumonia caused by COVID-19 [EB/OL], 2020. Available: http://www.nhc.gov.cn/xcs/zhengcwj/202001/e71c5de9 25a64eafbe1ce790debab5c6.shtml

14 Jiang W, Cao W, Liu Q. Wearing the N95 mask with a plastic handle reduces pressure injury. J Am Acad Dermatol 2020;82:e191-2.

15 Sim MR. The COVID-19 pandemic: major risks to healthcare and other workers on the front line. Occup Environ Med 2020;77:281-2.

16 National Health Commission of the People's Republic of China. Notice on further strengthening the protection of medical personnel during the epidemic prevention and control, 2020. Available: http:// www.nhc.gov.cn/yzygj/s7659/202002/75c6e88ecbeb42a9a26acb53 $8383 \mathrm{e} 2 \mathrm{fc} . \mathrm{shtml}$

17 Coleman S, Nixon J, Keen J, et al. A new pressure ulcer conceptual framework. J Adv Nurs 2014;70:2222-34.

18 Kayser SA, VanGilder CA, Ayello EA, et al. Prevalence and analysis of medical device-related pressure injuries: results from the International pressure ulcer prevalence survey. Adv Skin Wound Care 2018;31:276-85.

19 Song Z, Xie J, Zhu J. A "paper diaper" in N95 respirator. J Am Acad Dermatol 2020;Apr 29 S0190-9622:30745-3.

20 Smart H, Opinion FB, Darwich I, et al. Preventing facial pressure injury for health care providers adhering to COVID-19 personal protective equipment requirements. Adv Skin Wound Care 2020;33:418-27. 